\title{
Botox: information versus publicité
}

\author{
Comment les médecins spécialisés en médecine esthétique peuvent-ils informer au \\ sujet des traitements médicaux à base de toxine botulique (Botox) sans en faire la \\ publicité? S'appuyant sur l'arrêt rendu par un tribunal, Swissmedic est aujourd'hui \\ en mesure de publier les présentes directives.
}

Le Tribunal administratif fédéral confirme une décision rendue par Swissmedic au sujet de l'interdiction de faire de la publicité pour des médicaments dans le cadre de la promotion de prestations médicales (cf. arrêt passé en force de chose jugée du 17 octobre 2011; C-1795/2009 [1]). Les répercussions concrètes sont les suivantes:

Seules les informations générales sur la santé ou les maladies sont autorisées et n'entrent pas dans le champ d'application des dispositions relatives à la publicité énoncées dans le droit sur les produits thérapeutiques. En d'autres termes, de telles informations ne sont licites que dans la mesure où elles ne se réfèrent ni directement ni indirectement à des médicaments précis.

Pour que les sites web qui font de la publicité pour des prestations médicales soient conformes aux dispositions du droit sur la publicité de la législation sur les produits thérapeutiques, il faut notamment que le mot «Botox» ne soit pas utilisé pour désigner des applications spécifiques de médicaments contenant de la toxine botulique, car leur mention et représentation iconographique doivent être considérées comme de la publicité illicite pour des médicaments soumis à ordonnance.

Le mot «Botox» ne peut pas non plus être mis en évidence de quelque manière que ce soit; son utilisation en tant que titre ou dans un titre ou en intitulé de rubrique et/ou de sous-rubrique ainsi que dans un nom de domaine ou dans des adresses de sites Internet (chemin, URL) est illicite. Il est en revanche permis d'utiliser la désignation du principe actif «toxine botulique» dans des intitulés de rubriques ou pour l'explication du terme «traitement par le Botox» dans le corps d'un texte.

Pour que toutes les informations remplissent les critères d'exhaustivité, de pondération et d'objectivité, il est nécessaire de prendre en compte également les aspects suivants:

Correspondance: Swissmedic Hallerstrasse 7

CH-3000 Berne 9 Tél. 0313220276 Fax 0313220406

odette.klensch[at]swissmedic.ch

\section{Médicaments autorisés et indications autorisées pour le principe actif qu'est la toxine botulique}

$\mathrm{Au}$ total, trois préparations ayant pour principe la toxine botulique sont autorisées, mais une seule l'est pour un traitement qui peut être motivé aussi par des raisons esthétiques (traitement des rides glabellaires [aussi appelées «rides du lion»] modérées à graves chez les adultes). Et parce que les indications revendiquées auprès du public doivent correspondre à celles énoncées dans l'information sur le médicament, seule la mention du traitement des «rides du lion» est autorisée pour les traitements esthétiques à base de toxine botulique. Toutes les rubriques pertinentes d'un site web qui contiennent des informations sur des traitements à base de toxine botulique motivés par des raisons esthétiques ne peuvent par conséquent contenir que cette information et mentionner, le cas échéant, le traitement de l'hyperhidrose axillaire chez l'adulte, qui est une autre indication autorisée pour la toxine botulique.

\section{Off-label-use}

Dans le traitement des rides, la seule indication autorisée est le traitement des rides glabellaires («rides du lion»). L'utilisation de la toxine botulique pour estomper d'autres rides du visage (par ex. pattes d'oie, rides

\section{Dans le traitement des rides, la seule indication autorisée est le traitement des rides glabellaires («rides du lion»).}

du front, sillons naso-géniens, etc.) ou pour toute autre utilisation (par ex. migraines, céphalées par tension nerveuse, etc.) est donc considérée comme un «off-label-use», c'est-à-dire une utilisation d'un médicament en dehors des indications autorisées par Swissmedic. Le médecin traitant en assume seul l'entière responsabilité et il est tenu de dûment informer son patient/sa patiente.

Toute publicité pour des indications non autorisées est interdite. Il en va donc de même pour les renvois vers des sites web consacrés à de telles possibilités de traitement «off-label». Swissmedic accepte cependant le terme générique «rides d'expression» dans le corps des textes et l'énumération des rides à traiter 
(même de celles qui constituent un off-label-use) dans le cadre de propositions de prix, pour autant que cela soit fait avec retenue.

\section{Informations sur la sécurité}

Dans l'information sur le médicament autorisée par Swissmedic pour le traitement des rides glabellaires («rides du lion») par la toxine botulique, il est indiqué que d'après les résultats des essais cliniques, le taux de patients risquant de présenter un effet indésirable associé au traitement est de $24 \%$. Les possibles effets indésirables y sont par ailleurs classés selon leur probabilité de survenue: très fréquents $(>10 \%)$, fréquents $(1 \%-10 \%)$ et occasionnels $(0,1 \%-1 \%)$.

Toutes les rubriques pertinentes d'un site web doivent être conformes à l'information sur le médicament approuvée non seulement pour ce qui est des indications, mais également des informations sur la sécurité. Swissmedic recommande par conséquent d'inclure non seulement un passage (éventuellement synthétique) sur les risques liés à un tel traitement, mais également un lien vers la banque de données où sont regroupées les informations sur les médicaments telles qu'approuvées par Swissmedic.

\section{Tableau 1}

Mentions licites dans le cadre de la publicité pour des prestations médicales (traitements par la toxine botulique):

\begin{tabular}{ll} 
Indications & $\begin{array}{l}\text { Rides glabellaires («rides du lion») } \\
\text { Rides d'expression (sans énumération spécifique) } \\
\text { Hyperhidrose axillaire }\end{array}$ \\
\hline Effets indésirables & $\begin{array}{l}\text { Absolument indispensable pour une information factuelle } \\
\text { et mesurée; les informations doivent cependant concorder } \\
\text { avec l'information sur le médicament approuvée par } \\
\text { Swissmedic (information professionnelle) }\end{array}$ \\
\hline Début et durée de l'effet & $\begin{array}{l}\text { Ces informations doivent correspondre à celles figurant dans } \\
\text { l'information sur le médicament approuvée par Swissmedic } \\
\text { (information professionnelle) }\end{array}$ \\
\hline Toxine botulique & Mention du principe actif \\
\hline Traitement par le «Botox» & $\begin{array}{l}\text { La désignation «Botox» seulement en tant que description, } \\
\text { dans le corps du texte }\end{array}$ \\
\hline Information professionnelle & Mise en ligne
\end{tabular}

\section{Tableau 2}

Mentions illicites dans le cadre de la publicité pour des prestations médicales (traitements par la toxine botulique):

\section{Dénomination ou \\ représentation de médicaments \\ contenant de la toxine botulique}

Terme «Botox»

Dans tout le texte

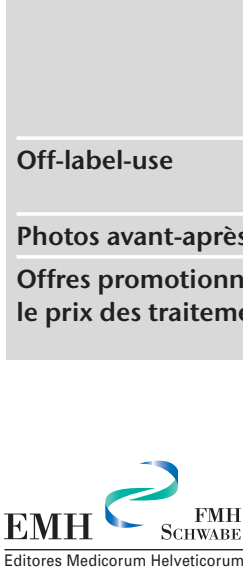

A l'intérieur du nom de domaine

Sur la page d'accueil de sites web

Comme titre d'une rubrique

Comme titre d'une sous-rubrique

A l'intérieur des adresses de sites Internet (chemin, URL)

Mentions telles que pattes d'oie, rides du front, sillons naso-géniens, rides labiales, rides du cou, migraines, etc. Considérées comme une promesse de résultat garanti

Incitation à un usage excessif

Offres promotionnelles affectant

ara
Informations sur le début et la durée de l'effet

De même, toutes les rubriques pertinentes d'un site web doivent contenir des informations sur le début et la durée de l'effet, qui doivent correspondre à celles figurant dans l'information sur le médicament telle qu'approuvée.

\section{Photos avant-après}

Les photographies et illustrations qui mettent en regard l'état avant et après le traitement doivent être considérées comme des représentations typiques de promesses quant à l'effet du médicament administré. Aussi ne sont-elles pas autorisées. Elles seraient d'ailleurs également illicites si les préparations à base de toxine botulique n'étaient pas soumises à ordonnance. Les photos avant-après sont dans tous les cas interdites sur les sites web.

\section{Offres promotionnelles affectant le prix du traitement par la toxine botulique}

Sont considérées comme des infractions particulièrement graves les offres promotionnelles consistant à accorder des remises sur des traitements à base de toxine botulique. Ce type d'offre contrevient à l'interdiction de la publicité destinée au public et est constitutif d'une publicité pour un médicament susceptible de conduire à un usage excessif, abusif ou inapproprié et, partant, de mettre en danger la santé du patient.

Les offres promotionnelles sur les traitements esthétiques à base de toxine botulique sont donc interdites et font l'objet de poursuites pénales engagées par Swissmedic.

\section{Références}

1 www.bvger.ch/publiws/pub/search.jsf $\rightarrow$ dans le champ «Texte de recherche», saisir C-1795/2009 et cliquer sur «rechercher».

- Loi sur les produits thérapeutiques (LPTh): Loi fédérale du 15 décembre 2000 sur les médicaments et les dispositifs médicaux (Loi sur les produits thérapeutiques, LPTh; RS 812.21) www.admin.ch/ch/f/ rs/c812 21.html

- Message concernant une loi fédérale sur les médicaments et les dispositifs médicaux (LPT): www.amtsdruckschriften.bar.admin.ch $\rightarrow$ saisir le mot-clé «LPTh» ou accès direct: www.amtsdruckschriften.bar.admin.ch/viewOrigDoc. do?id=10055092

- Ordonnance sur la publicité pour les médicaments (OPMéd): Ordonnance du 17 octobre 2001 sur la publicité pour les médicaments (Ordonnance sur la publicité pour les médicaments, OPMéd; RS 812.212.5). www.admin.ch/ch/f/rs/c812_212_5.html

- Loi sur les professions médicales (LPMéd): Loi fédérale du 23 juin 2006 sur les professions médicales universitaires (Loi sur les professions médicales, LPMéd; RS 811.11). www.admin.ch/ch/f/rs/c811_11.html

- Tribunal administratif fédéral; arrêt C-1795/2009. www.bvger.ch/publiws/pub/search.jsf $\rightarrow$ dans le champ «Texte de recherche», saisir C-1795/2009 et cliquer sur «rechercher». 